\title{
Peccati e peccatori
}

\section{Paola Vecchi Galli}

Università di Bologna

\begin{abstract}
Il tema del peccato è tanto diffuso nell'opera volgare e latina di Petrarca da rendere necessario un energico taglio interpretativo, una qualunque delimitazione di campo. Proprio questa ampiezza è però il segno di un eclettismo che mette a confronto linguaggi e specialismi diversi, a volte implicati fra loro sino alla reciproca indistinzione. Quanto segue è un primo percorso fra i peccati di Petrarca, con soste che testimoniano livelli diversi di esercizio delle parole del peccato: un primo livello, interdiscorsivo, di appropriazione terminologica all'interno di un orizzonte, quello lirico del Canzoniere, che lascia affiorare il tema della colpa correlandolo alla fabula dell'amore; e un secondo livello, di sviluppo di campi semantici legati al tema del peccato, sullo sfondo delle opere latine.
\end{abstract}

Parole chiave: Petrarca, Canzoniere, peccato, passioni, remedium.

\section{Abstract}

The topic of sin is so widespread in the vulgar and Latin work of Petrarch that it requires energy with an interpretative edge, one without delimitation of field. This expanse alone is but a sign of an eclecticism which draws comparisons between diverse languages and specialisms, occasionally intertwined to the point of reciprocal indifference. The following is an early journey through the sins of Petrarch, with breaks which witness the different levels of exercise of the words of sin: first, an interdiscursive level on the terminological appropriation within a horizon, such as the verse of the Canzoniere, which delved into the emotion of guilt, in correlation with the fable of love; and second, on the development of semantic fields in relation to sin, on the backdrop of Latin works.

Key words: Petrarch, Canzoniere, sin, passion, healing. 


\section{Per cominciare: la saligia}

Petrarca scrive molte volte il suo peccato. Poeta et historicus, ${ }^{1}$ è anche uomo di chiesa e di intime lacerazioni: da questa sua familiarità con il peccato gli provengono gesti minuti e quotidiani (come quando legge l'Ufficio nel suo Breviario, compone la nota dei peccati e le preghiere, stila elenchi sibillini di cadute nella colpa). Sarebbe perciò riduttivo circoscrivere il suo peccato alle cathene di cui fa espressamente ammenda nel Secretum, al factum illud obscenum che è stato per lui lo stimolo del sesso, all'indignantissimus animus che lo inclina all'ira (Posteritati, 8-9). La cifra del peccato si espande oltre questi luoghi e queste confessioni, sino a comporre un tessuto grandioso «intrecciato di fili multicolori»: un ambito che comprende i trattati religiosi e i "breviari laici» della mutatio vite (il De remediis utriusque fortune, secondo la definizione di Michele Feo), l'epistolario, le postille, le opere volgari. Impossibile quindi parlarne in forma sistematica. La parola, il tema, l'orizzonte concettuale e linguistico della colpa sono diffusi ovunque nella sua opera, tanto da rendere necessario un energico taglio interpretativo, una qualsiasi riduzione di campo.

Il peccato di Petrarca non può d'altronde prescindere dal tempo che lo produce: misurandosi da un lato con il terreno, prossimo all'antropologia, della fede collettiva e popolare, del terrore della morte e dell'endemico senso di colpa, ${ }^{2}$ rielaborato dalla pastorale, dalla tradizione figurativa e dalla poesia popolareggiante (in specie quella fondata sulla catena acrostica della saligia); dall'altro con la teologia e con l'altissima letteratura religiosa (Dante in testa). Non c'è da stupirsi, quindi, che nel Canzoniere alcuni componimenti siano in grado di reagire con la letteratura del peccato contemporanea, anche quella popolareggiante.

I sette peccati capitali sono stati fatti oggetto, nel Trecento, di una curiosa e, col senno di poi, in fondo bonaria poesia gnomica in volgare: quella che, ad esempio nei sonetti di Fazio degli Uberti, dà origine alla catena della saligia (un aiuto della memoria in forma di acronimo ben chiaro al lettore del tempo, che si forma con le lettere iniziali di superbia-avarizia-lussuria-ira-gola-invi-

1. Ricorda e illustra la definizione (poeta e historicus) che di se stesso Petrarca dà a partire dal Laureae Privilegium del 1341 (con le implicazioni che i due termini poeta e historicus reciprocamente comportano) M. FEO, «Petrarca e Boccaccio: critica e filologia», in E. MALATO (dir.), Storia della letteratura italiana, vol. XI, La critica letteraria dal Due a Novecento, Roma: Salerno Editrice 2003, p. 103-29, a p. 103.

2. È uno stilema usato da J. DelumeaU, Il peccato e la paura. L'idea di colpa in Occidente dal XIII al XVIII secolo, Bologna: Il Mulino, 1987 [1983], che avrò modo di citare anche in seguito. Il terrore dell'inferno è tema già colto da De Sanctis nello Specchio di Penitenza del coetaneo di Petrarca Jacopo Passavanti: «La musa del Passavanti è il terrore, e la sua materia è il vizio e l'Inferno [...]. Diresti che provi voluttà a spaventare e tormentare l'anima». Esplora la medesima temperie spirituale, dominata dalla paura del castigo eterno, anche il vecchio (ma ancora utile) volume di A. LEVASTI, Mistici del Duecento e del Trecento, Milano-Roma: Rizzoli, 1935. Sulla stessa lunghezza d'onda di De Sanctis il suo giudizio lapidario sul Passavanti: "Col Passavanti tutto è tetro e terribile [...]. Il suo uomo è il perpetuo peccatore che sta sotto il continuo incubo di un aspro e duro giudizio, e l'unica salvezza, in terra, è la perpetua penitenza» (ibid., p. 64). 
dia-accidia). ${ }^{3}$ La saligia in rima può essere percepita come una deriva "teologica» e poetica della grande costruzione morale di Dante e della trattistica duecentesca sul peccato, una sorta di concentrato dell'Inferno in forma di proverbia, un prodotto di recupero derivato dalle frattaglie più memorabili della Commedia e della predicazione. Quanto in Dante è potente costruzione dello spirito si fa gusto popolareggiante e sentenzioso nei componimenti di Fazio e dei suoi epigoni. Sono litanie prossime ai cartigli e alle epigrafi che ornano gli affreschi all'insegna del "visibile parlare», ${ }^{4}$ imparentate con la devozione collettiva, piuttosto che con la teologia morale.

3. I sonetti sui peccati capitali di Fazio degli Uberti si leggono nei Rimatori del Trecento, (a cura di G. CORSI), Torino: UTET, 1969, p. 275-276 (con numerosi rinvii ad altra poesia coeva sul tema dei peccati capitali). Se ne vedano alcuni capoversi (I'son la gola che consumo tutto, I' son la mala pianta di superba, I'son la scellerata de lussuria) in B. BENTIVOGLI, P. VECCHI GALli (a cura di), IUPI, Incipitario unificato della poesia italiana, vol. III, Edizioni di lirica antica, Modena: Franco Cosimo Panini, 1990, ad indicem. La catena, poetica e dottrinale, della saligia, ben nota ai frequentatori di rime morali del Tre-Quattrocento, è bevemente illustrata (con bibliografia pregressa e numerosi rinvii a componimenti frottolistici sui vizi e i peccati capitali) da S. VERHULST, La frottola (XIV-XV Sec.): aspetti della codificazione e proposte esegetiche, Gent: Rijksuniversiteit te Gent, 1990, in part. p. 61-91. Da ultimo la saligia è sommariamente citata da J. BASCHET, «I peccati capitali e le loro punizioni nell'iconografia medievale», in C. CASAGRANDE-S. VECCHIO, I sette vizi capitali. Storia dei peccati nel Medioevo, Torino: Einaudi, 2000, p. 239, e da F. RigOTTI, La filosofia in cucina, Bologna: Il Mulino, 2004, p. 92-93.

4. In cui anche Petrarca ebbe voce in capitolo, se è vero che prese attivamente parte al progetto degli affreschi della Sala dei Giganti di Padova. Sul visibile parlare e le sue varie accezioni (come l'incrocio fra immagini e testualità, una sorta di pastorale figurata espressa in un linguaggio stereotipo), la bibliografia è ormai amplissima e di alto livello teorico e documentario. Il motivo di tanto interesse per le scritture esposte va ricercato nella possibilità di leggere in esse non solo una forma di deriva della grande letteratura religiosa coeva, ma anche uno strumento multimediale (da affiancare alla predicazione) di diffusione spirituale e letteraria. Mi limito qui a citare le voci più utili al mio discorso (a partire dalle prime indagini della Scuola Storica sino agli ultimi aggiornamenti della critica): S. MORPURGO, «Le epigrafi volgari in rima del "Trionfo della Morte", del "Giudizio universale e Inferno" e degli "Anacoreti" nel Camposanto di Pisa", L'Arte, n. 2, 1899, p. 51-87; L. BATTAGLiA RICCI, Ragionare nel giardino. Boccaccio e i cicli pittorici del "Trionfo della morte», Roma: Salerno Editrice, 1987; ID., Parole e immagini nella letteratura italiana medievale. Materiali e problemi, Pisa: Gei, 1994; M. CiccuTO, Limmagine del testo. Episodi di cultura figurativa nella letteratura italiana, Roma: Bonacci, 1990; C. Ciociola (a cura di) "Visibile parlare». Le scritture esposte nei volgari italiani dal Medioevo al Rinascimento, Napoli: ESI, 1995; C. Ciociola, «Scrittura per l'arte, l'arte per la scrittura», in E. Malato (dir.) Storia della letteratura italiana, III, Il Trecento, Roma: Salerno Editrice, 1995, p. 531-580 (anche per i rinvii alla letteratura secondaria); J. BASCHET, op. cit.; M. L. MENEGHETTI, «La cultura visiva (affreschi, rilievi, miniature)», in P. BoITANI, M. MANCINI, A. VÀrVARO (dir.) Lo spazio letterario del Medioevo, 2. Il Medioevo volgare. La circolazione del testo, Roma: Salerno Editrice, 2002, p. 463-488; L. BOLZONI, La rete delle immagini. Predicazione in volgare dalle origini a Bernardino da Siena, Torino: Einaudi, 2002; ID., "Predicazione in volgare e uso delle immagini, da Giordano da Pisa a Bernardino da Siena», in G. AuZZAS, G. BAFFetTI, C. DELCORNO (a cura di), Letteratura in forma di sermone. I rapporti tra predicazione e letteratura nei secoli XIII-XVI, Firenze: Olschki, 2003, p. 29-52. 


\section{Peccati nel Canzoniere}

Anche Petrarca non ne è immune, almeno a leggere il sonetto 232 del Canzoniere, una sorta di minimo "trionfo dell'ira», impastato di erudizione mitologica e storica, quasi un omaggio (o una sfida) alla saligia dei poeti minori del suo tempo:

Vincitore Alexandro l'ira vinse, et fe' 'I minore in parte che Philippo: che li val se Pyrgotile et Lysippo l'intagliâr solo et Appelle il depinse?

Lira Tydëo a tal rabbia sospinse, che, morendo ei, si róse Menalippo; l'ira cieco del tutto, non pur lippo, fatto avea Silla: a l'ultimo l'extinse.

Sa 'I Valentinïan, ch'a simil pena ira conduce: et sa 'l quei che ne more, Aiace in molti, et poi in se stesso, forte.

Ira è breve furore, et chi nol frena, è furor lungo, che 'l suo possessore spesso a vergogna, et talor mena a morte.

Furore (in Eccli. 27 33: «Ira et furor, utraque execrabilia sunt»), ma anche giusto sdegno (zelo), come quello esercitato da Gesù contro i mercanti nel Tempio - tale è l'ira del ciel di RVF 24, 2, o l'ira di Dio di RVF 137, 2-, l'ira è giunta a Petrarca carica di echi e di significati del passato: che comprendono l'ira aristotelica, naturale e positiva, la dantesca ira mala (di derivazione senecana), e l'ira, che è giustizia, di Dio. ${ }^{5}$ E dunque Petrarca si inserisce in un coro che abbraccia almeno la Bibbia, Aristotele e Cicerone, Orazio, Seneca, San Gerolamo e Dante.

Su tali fondamenti, nel sonetto 232 del Canzoniere l'ira non può se non essere descritta secondo un disegno all'apparenza semplice, ma scientifico e dottrinale nella sostanza, che si apre, come un repertorio di esempi (o come una sequenza di aneddoti spirituali), con una casistica di eroi travolti da questo peccato. È fuor di dubbio che, come ha notato Natascia Tonelli, il sonetto rinvii "prepotentemente all'ambito "naturale" ", evocando soprattutto, con la parte medica dello Speculum doctrinale di Vincent de Beauvais, la fisiologia di una passione che ha precisi connotati fisici: il rossore, il furore, l'accecamento improvviso, e di conseguenza la pazzia e talora la morte. ${ }^{6}$ D'altronde,

5. Per una trattazione succinta del vizio dell'ira, e dei suoi connotati dottrinali, filosofici e letterari, si può ricorrere anche al confronto con l'universo dantesco, su cui cfr. in particolare P. Boyde, "L'ira», in ID., "Lo color del core». Visione, passione e ragione in Dante, Napoli: Liguori, 2002, p. 275-307, e la voce «Ira» di F. Montanari per l'Enciclopedia dantesca, III, Roma: Istituto della Enciclopedia italiana, 1971, p. 513-516.

6. Cfr. N. TonelLI, «Elementi di cultura medica nei "Rerum vulgarium fragmenta”, Quaderni petrarcheschi, n. 11, 2001, p. 228-51. 
Petrarca dà prova di simile riflessione sull'ira anche in Secretum II 122 e s.; e più tardi anche il De remediis II 107 richiamerà con una battuta fulminea la pazzia dell'iracondo: "Dolor// Ira estuo. / Ratio// Furis ergo".

Ma non basta. Esplorati gli aspetti filosofici e patologici dell'ira, Petrarca dà rilievo a un aspetto dell'ira su cui anche filosofia e teologia si sono a lungo interrogate. ${ }^{7}$ Due linguaggi specialistici collidono: servendosi di parole all'apparenza semplicissime, Petrarca punta al nucleo teologico-morale di questo vizio-passione, distinguendo l'ira breve - passione istintiva dell'uomo, incontenibile e di per sé non censurabile- dall'ira lunga, che diviene, se non è tenuta saldamente sotto controllo (chi nol frena), peccato capitale proprio «in ragione di due elementi: il suo oggetto e la sua intensità». ${ }^{8}$ Il riferimento all'ambito del peccato si fa più trasparente: bastano tre versi (12-14), come il cartiglio di un affresco, per riassumere il perno spirituale attorno a cui ruota il sonetto. Implicati fra loro, ipotesti classici, ${ }^{9}$ episteme scientifica e aspetti della riflessione cristiana attorno all'ira danno senso e nutrimento morale alle figure «in rosso» (il colore di questo peccato: Alessandro, Tideo, Silla, Valentiniano, Aiace) che popolano l'immaginario erudito di Petrarca. Quasi con le stesse parole Petrarca riproporrà il tema nel già citato capitolo De ira del De remediis utriusque fortune (II 107), sottolineando fra tutte la sola fonte oraziana: ${ }^{10}$

Ratio// Furis ergo.

Ita furor brevis est,

inquit Flaccus. Fit autem a plerisque mala consuetudine et impatientia longus furor. [...] Hoc horumque similia frenare iram debent, ne sequaces suos, ut solita est, in infamiam clademque precipitet.

L'episodio, per quanto minimo, è, a mio parere, esemplare. È difficile riportare su un'unica linea maestra, linguistica e teologica, la percezione petrarche-

7. Richiamandosi a sua volta in particolare alla tradizione aristotelica (Et. Nic.) e stoica (il De ira senecano).

8. Come in San Gerolamo (Epist. 79 a Salvina) che aveva ammonito: «irasci hominis est, iram non perficere Christiani». Sul rapporto ira breve-ira lunga cfr. soprattutto C. CASAGRANDE - S. VECCHIO, op. cit., p. 68: ma è importante l'intero paragrafo Adirarsi non sempre è peccato, ibidem, p. 66-70.

9. Fra i quali Marco Santagata (nel suo commento a Francesco Petrarca, Canzoniere, Milano: Mondadori, 1996, n. 232, p. 955) segnala appunto il De ira senecano e il pertinentissimo Orazio di Epist. I 2, 62 (ira furor brevis est), ricalcato alla lettera da Petrarca anche in De remediis II 107.

10. Per il testo e il commento di De remediis rinvio a C. CARRAud (a cura di), Les remèdes aux deux fortunes, De remediis utriusque fortune, (Préface de G. ToGNON, Introduction, notes et index par C. Carraud), Grenoble: Millon, 2002, II p. 613-14 (mio il corsivo). Ma sul passo è fondamentale anche M. FEO «Petrarca» in Orazio, Enciclopedia oraziana III, Roma: Istituto della Enciclopedia italiana, 1998, p. 405-425, seguita da A. La PENNA, «Una nota su Petrarca e Orazio», Quaderni petrarcheschi, n. 11, 2001, p. 163-166. 
sca del peccato. ${ }^{11}$ Non riducibile alla sola norma cristiana, essa ci permette però, fin da questo episodio, di marcare l'importanza di concetti come il freno, o il rationis frenum: ${ }^{12}$ la riflessione sul peccato è soggetta anche alla misura della filosofia stoica (e alla casistica connessa delle passioni).

E l'ira è il vizio più praticato (almeno di nome) nel Canzoniere. Mentre da un lato essa porta a vergogna e a morte colui che se ne è reso colpevole (nel campo concettuale che si è instaurato nel sonetto 232, intenderei piuttosto la morte dell'anima che quella del corpo), altrove il valore del vocabolo è però semanticamente più sfumato. In $R V F 332,14$, ad esempio, Petrarca si chiede: "Ove è condutto il mio amoroso stile? / A parlar d'ira, a ragionar di morte», mutuando la parola del peccato (o della passione) per esprimere la sua «angoscia» amorosa (e di certo il sintagma risente delle accezioni cortese o stilnovistica, opponendo in questi termini la donna angelicata alle comuni femmine: "ogn'altra ver' di lei i' la chiam' ira»). ${ }^{13}$ Più pertinente, invece, l'ira di cui si dichiara affetta Laura in TM II, 100-102 ("più di mille fiate ira dipinse / il volto mio, ch'Amore ardeva il core; / ma voglia, in me, ragion già mai non vinse»): che è appunto lo sdegno che signoreggia la volontà.

Il sonetto 232 resta perciò solo a testimoniare l'attenzione di Petrarca a una moda quasi folclorica: il poeta è infatti lontano dal trasformare i Fragmenta in aneddoti, sentenze e medaglioni esemplari, buoni per le summae dei predicatori. ${ }^{14}$ Così come è decisivo che non vi ricorrano, per quel che ho potuto verificare, altri elenchi di peccatori: il piccolo drappello di iracondi resta isolato a rappresentare le «schiere del peccato", a cui Dante aveva invece tanto spesso fatto ricorso nella Commedia.

Se ne ha conferma in un altro sonetto ( $R V F$ 172), dove Petrarca stempera nel contesto amoroso il timbro epigrammatico dei versi iniziali, aperti dalla parola dell'invidia:

\section{O Invidia nimica di vertute,}

ch'a' bei principii volentier contrasti, per qual sentier cosí tacita intrasti in quel bel petto, et con qual' arti il mute?

Da radice n'ài svelta mia salute: troppo felice amante mi mostrasti a quella che' miei preghi humili et casti gradí alcun tempo, or par ch'odi et refute.

11. Né mi risulta che siano stati operati finora tentativi sistematici di esplorazione del mondo del peccato petrarchesco, se si eccettua il recente esame di Ilaria Tufano, «La notte, la paura, il peccato. Il ritratto dell'occupatus nel De vita solitaria», Rassegna europea di letteratura italiana, n. 22, 2003, p. 37-52.

12. La categoria di freno della ragione è ovviamente funzione variabile dell'importanza che si assegna al peccato. Le componenti di fondo di un linguaggio di questo tipo derivano in Petrarca da fonti stoiche e senecane.

13. Si tratta del sonetto di Guido Cavalcanti Chi è questa che vèn, chóogn'om la mira, v. 8.

14. Come sono riportati, a esempio, in G. VARANINI E G. BALDASSARRI (a cura di), Racconti esemplari dei predicatori del Due e Trecento, Roma: Salerno Editrice, 1993. 
Né però che con atti acerbi et rei del mio ben pianga, et del mio pianger rida, poria cangiar sol un de' pensier' mei; non, perché mille volte il dí m’ancida, fia ch'io non l'ami, et ch'i' non speri in lei: che s'ella mi spaventa, Amor m'affida.

La casistica dell'invidia è qui pretesto non per una trattazione dottrinale, ma per una breve divagazione di gusto erotico-cortese. ${ }^{15}$ Ciò non toglie che la succinta descrizione di Laura invidiosa racchiuda, almeno in accenno, alcuni dei connotati psico-patologici del peccato (l'amore traformato in odio, l'aspetto minaccioso della persona). Ma sono soprattutto l'infelicità per la fortuna e il dolore per il bene altrui i marcatori dell'invidia "cristiana" (e - a ben guardare- i fili conduttori sotterranei del componimento), giusta l'avvertenza di Peraldo (II, p. 349): «Possumus distinguere duas species invidiae, scilicet exultationem in adversis proximi, et tristitiam in prosperis». ${ }^{16} \grave{\mathrm{E}}$ un aspetto della riflessione sull'invidia che - diventato quasi senso comune- chiama però in causa ipotesti remoti, come il Gregorio Magno del Regulae pastoralis liber:

Gli invidiosi si esorteranno a riflettere seriamente quanto sia disdicevole la loro cecità nel rattristarsi dell'altrui profitto e nello struggersi perché altri godono. Che miseria è codesta di taluni che diventano peggiori, perché gli altri migliorano e, vedendo prosperare il prossimo, si rodono di invidia fino a restar vittime di questa peste del cuore. Chi più infelice di costoro che la vista della felicità altrui rattrista d'una pena che li rende più colpevoli? Se amassero quel bene che vedono negli altri e non possono avere, in certo modo l'amore glielo farebbe possedere. ${ }^{17}$

D'altronde, l'attenzione di Petrarca alla semantica dell'invidia non si esaurisce in queste battute: punto sul vivo dagli strali polemici dei detrattori (i quattro aristotelici veneziani), nel De sui ipsius et multorum ignorantia (seguito dalla Sen. V 2) egli darà dell'invidia una descrizione espressiva e grottesca (tutta giocata all'interno del campo semantico dei morsi dei «cani di Averroè»), sul filo delle indicazioni dottrinali di Gugliemo Peraldo e di Pietro Bersuire. Il tema dell'invidia esulerà allora dalla fruizione della breve tessera linguistica,

15. Di cui Petrarca dà un'altra prova in $R V F 130,12-14$. D'altronde, l'invidia è peccato sociale per eccellenza, di uomini di corte e intellettuali, come già in Dante, Inf. XIII, 64-66 (non il primo a trattare di questo peccato, la cui casistica rimonta al solito Gregorio Magno): cfr. la voce "Invidia» di F. SALSANO nella Enciclopedia dantesca, cit. III, 1971, e C. CASAGRANDE - S. VECCHIO, op. cit., p. 38-43.

16. Sulla casistica dell'invidia è utile richiamare anche il testo di De remediis II 106 (in C. CARRAUD op. cit. I p. 1004-1005): De invidia active), in cui Ratio ricorda a Dolor che l'invidia, a differenza degli altri vizi, solis malis pascitur et torquetur bonis (e cfr. anche il commento in ibid., II, p. 612, da cui è tratta la citazione di Peraldo, Summae virtutum ac vitiorum Tomus secundus, riportata a testo).

17. La citazione è tratta da Gregorio MAGNO, «La regola pastorale», in E. ZoLLA, I mistici dell'Occidente, Milano: Adelphi, 1997, I, p. 508. 
per acquisire la complessità di un più ampio discorso spirituale (contro l'aridità culturale dei dialettici), in quel caso arricchito da precisi apporti della tradizione iconografico-teorica cristiana. ${ }^{18}$

Questi esempi, ricavati dal Canzoniere e dall'opera latina di Petrarca, non sono che episodi minimi, la punta dell'iceberg di un'impressionante letteratura del peccato, in latino e in volgare, in forma di poesia, di summa, di ars praedicandi, di trattato, trattatello e di enciclopedia dei vizi e delle virtù, di bestiario, di manuale per la confessione e per la penitenza, ${ }^{19}$ che permea la letteratura dell'Occidente nell'autunno del Medioevo. Petrarca ne è —a vario titolo- partecipe, in poesia volgare e in prosa latina. ${ }^{20}$ Il campo semantico del peccato (nei suoi molti aspetti formali e nuclei narrativi: lessicale o tematico; e ancora, dottrinale, penitenziale, confessionale) è una langue, o una grammatica, che inonda la sua opera, in modi ora allusivi e sfumati, ora puntuali e complessi, attingendo piuttosto all'ambito dell'interdiscorsività che a quello dell'intertestualità. ${ }^{21}$

Eppure, questa lingua è assente in luoghi dove ci aspetteremmo di rintracciarne attestazioni ampie. Penso ad esempio ai Triumphi, se è vero che su

18. Ad esempio, con l'immagine della rabida invidia, e degli invidiosi come cani latranti. Dell'invidia, subita e descritta da Petrarca, ha dato un quadro teorico e analitico interessante (illustrandone i campi metaforici e l'immaginario), C. POLITO, "Inter cunctas eminens obliqui causa iudicii livor". Annotazioni in margine al De ignorantia petrarchesco", Studi e problemi di critica testuale, n. 41, 1990, p. 5-28 (anche con qualche rinvio alla poesia volgare petrarchesca).

19. Oltre che C. CASAgrande - S. Vecchio, op. cit., è utile anche la sintesi «Peccato», composta dalle stesse autrici per J. LE GOFF e J.-C. SCHMITT (a cura di), Dizionario dell'Occidente medievale. Temi e percorsi, Torino: Einaudi, 2004, vol. II, p. 871-884. Per la concezione dantesca del peccato (con altra bibliografia, applicabile anche all'universo petrarchesco), cfr. P. DelHaYe, «Peccato», in Enciclopedia dantesca, cit. IV, 1973.

20. E, anzi, ci sarà piuttosto da meravigliarsi che fino a oggi non si sia tentata una ricognizione sistematica di questo amplissimo ambito dottrinale, tematico, linguistico e semantico, che inerisce al tema, ancora più vasto, della religiosità petrarchesca. Più sviluppata è invece la pista che tratta del contenuto religioso e penitenziale del Canzoniere: per la quale sono particolarmente importanti i vecchi studi di M. CASALI, "Petrarca "penitenziale": dai "Salmi" alle "Rime" ", Lettere Italiane, n. 20, 1968, p. 361-381, e B. MARTINELLI, «L'ordinamento morale del Canzoniere del Petrarca" [1976], poi in ID., Petrarca e il Ventoso, Bergamo: Minerva Italica, 1977, p. 217-300. Così come è oggetto di una ricchissima bibliografia l'agostinismo del Petrarca: per il quale mi limito a rinviare ai saggi più recenti: C. CALCATERRA, Sant'Agostino nelle opere di Dante e del Petrarca, Milano: Vita e Pensiero, 1931; P. P. GEROSA, Umanesimo cristiano del Petrarca. Influenza agostiniana, attinenze medievali, Torino: Bottega d'Erasmo, 1966; É. LuCIANI, Les Confessions de saint Augustin dans les lettres de Pétrarque, Paris: Études Augustiniennes, 1982; G. POzzI, Petrarca, i Padri e soprattutto la Bibbia [1989], poi in ID., Alternatim, Milano: Adelphi, 1996, p. 143-189; E. GIANNARELLI, «Petrarca e i Padri della Chiesa", Quaderni petrarcheschi, n. 10, 1991, p. 393-412; R. BETTARINI, "Fluctuationes" agostiniane nel "Canzoniere" di Petrarca", Studi di Filologia Italiana, n. 60, 2002, p. 129-39. M. SANTAGATA, I frammenti dell'anima. Storia e racconto nel Canzoniere di Petrarca, Bologna: Il Mulino, 2004, passim.

21. Nel corso del Convegno ho dato brevemente ragguaglio anche delle componenti dottrinali dei sonetti 6 e 7 dei $R V F$. Per motivi di opportunità editoriale non è stato qui possibile riprendere per intero quelle pagine, che svilupperò in un mio prossimo intervento sul tema. 
un'impostazione dottrinale hanno la meglio l'erudizione classica e la fenomenologia delle passioni: gli amanti del primo trionfo (Triumphus Cupidinis) o gli uomini illustri del quarto (Triumphus Fame) potrebbero essere, ma non sono, peccatori di lussuria o di vanagloria (sono invece amorosa greggia e onorata gente); la loro punizione potrebbe essere, ma di fatto non $\grave{e}$, punizione dei peccati. Non stupisce perciò che il poema eluda la dimensione cristiana della colpa e della pena esorcizzando del tutto la parola del peccato. ${ }^{22}$ Questa la descrizione di Amore, dei suoi prigionieri e del suo carcere:

d'intorno innumerabili mortali, parte presi in battaglia, e parte occisi, parte feriti di pungenti strali (TC I, 28-30);

Questi è colui che 'l mondo chiama Amore: amaro, come vedi, e vedrai meglio quando fia tuo, com'è nostro signore.

Giovencel mansueto, e fiero veglio: ben sa chi 'l prova, e fiate cosa piana anzi mill'anni; infin ad or ti sveglio.

Ei nacque d'ozio e di lascivia humana, nudrito di penser dolci soavi, fatto signore e dio da gente vana (TC I, 86-94);

In così tenebrosa e stretta gabbia rinchiusi fumo, ove le penne usate mutai per tempo e la mia prima labbia;

e 'ntanto, pur sognando libertate, l'alma, che 'l gran disio fea pronta e leve, consolai col veder le cose andate.

Rimirando er' io fatto al sol di neve tanti spirti e sì chiari in carcer tetro, quasi lunga pictura in tempo breve, che 'l pie' va inanzi, e l'occhio torna a dietro (TC IV, 157-166).

Mentre questa è la riflessione sulla Fama:

[...] mirando intorno su per l'erba, vidi da l'altra parte giugner quella che trae l'uom del sepolcro e 'n vita il serba. [...] scolpito per le fronti era il valore de l'onorata gente [...] (TF III, 7-9; 19-20).

22. Peccatore è una voce che non compare mai nei Triumphi: le schiere che compongono le processioni trionfali non hanno quindi come immediato referente una dimensione religiosa o morale. Una sola volta ricorre peccato («e quei che cominciò poi la gran torre / che fu sì di peccato e d'error carca») in Triumphus Fame II, 80-81, con allusione a Nembrot e alla costruzione della torre di Babele, non a caso simbolo di superbia e principio della confusione delle lingue degli uomini. 
Ma per la turba, a' grandi errori avezza, dopo la lunga età sia il nome chiaro: che è questo però che sì s'apprezza?

Tanto vince e ritoglie il Tempo avaro; chiamasi Fama, ed è morir secondo, né più che contra ' 1 primo è alcun riparo.

Così il Tempo triompha i nomi e 'l mondo! (TT, 139-145).

È impossibile leggere questi versi senza pensare che il contromodello dantesco abbia agito in Petrarca come deterrente, distogliendolo una volta per tutte, nei Triumphi, da campi semantici della colpa e della pena troppo segnati da quelli della Commedia. ${ }^{23}$ Siamo davvero agli antipodi di Dante, e all'interno di una percezione dell'amore e della fama tutta legata all'umano. D'altronde, l'unica penitentia evocata nel poema è quella, terrena, di TC IV, 119, inserita all'interno della lunga digressione allegorica del trionfo d'Amore («dubbia speme davanti e breve gioia,/ penitentia e dolor dopo le spalle»).

\section{Remedium e sapientia}

In accezioni articolate ma anche - lo abbiamo visto - con clamorose assenze, nella sua opera Petrarca delinea il paesaggio della colpa non solo attingendo alla tradizione spirituale e dottrinale del suo tempo, ma anche al mondo classico - poetico e erudito - o a quello scientifico, filosofico e naturale della sua contemporaneità. La linea maestra del peccato, l'andamento argomentativo di fondo sono segnati da campi concettuali in grado, meglio di altri, di raccogliere e, se è possibile, armonizzare le fila di un pensiero onnivoro e inquieto. Che il peccato di Petrarca possa riconoscersi in primo luogo nella parola di Agostino e nella grande — o minuta — letteratura spirituale e pastorale del suo tempo, dai Padri ai mistici ai manuali dei predicatori, è fuor di dubbio: ma anche la concezione medica e farmacopeica delle passioni e delle affezioni fisiche a esse correlate spesso è tutt'uno con quella del peccato. ${ }^{24}$ L'osservazio-

23. Non voglio sostenere che il poema non sia permeato da una profonda visione di fede, per di più arricchita da una sostanza teologica attuale (come il grande tema, dibattuto anche in sede papale, della visio beatifica): esito un po', tuttavia, a vedere nei Triumphi solo un «modello di poesia cristiana», come sembra sostenere anche C. BERTOLANI, Il corpo glorioso, Roma: Carocci, 2001, p. 64. Semmai, occorrerà distinguere fra trionfo e trionfo, e leggere in chiave spirituale soprattutto i capitoli della Morte e della Eternità.

24. Fra le tante possibili connessioni e interrelazioni testuali, mi piace richiamare La medicina dell'anima (2) di Ugo di San Vittore, che collega i quattro umori del corpo e gli elementi e le stagioni del creato alle condizioni del peccato: «L'animo umano adopera quattro umori [del pari di quattro elementi si compone il mondo]: come sangue la dolcezza, come bile rossa l'amarezza, come bile nera la tristezza, come linfa la serenità della mente. [...] Nella contemplazione con la dolcezza dev'esserci anche l'amarezza per il ricordo del peccato, la tristezza per la sua esecuzione, la serenità per l'emendamento. Anche si deve badare a che la dolcezza spirituale non sia turbata dall'amarezza temporale, o che l'amarezza avuta dal peccato non si corrompa per la dolcezza carnale, che l'utile tristezza non sia turbata da ignavia, o che la mente serena non sia disciolta da cose illecite. L'anima ha anche lei le sue stagioni, 
ne non è scontata se ripensiamo all'ininterrotta polemica di Petrarca contro i medici e contro la pseudo-scienza dei contemporanei, a vantaggio di una sapientia superiore dell'uomo. ${ }^{25}$ Verrebbe quasi da pensare che il frequente ricorrere a termini come medicus, remedium e salus (in luogo di sanitas), abbia proprio lo scopo di risemantizzare in senso morale l'ambito della medicina. In questa reinterpretazione, omnipotens medicus è Dio, il solo in grado di sanare il cecum animi vulnus del peccatore Petrarca (Fam. X 5, 27, al fratello Gherardo).

Si rifletta su un caso esemplare. Se alla malattia del corpo si oppone tradizionalmente il remedium (la parola ha il significato tecnico della moderna medicina), ${ }^{26}$ sono innumerevoli i luoghi petrarcheschi - a partire dal Secretum e dal De remediis utriusque fortune - dove questo vocabolo sfuma decisamente verso un soprasenso morale e spirituale. ${ }^{27} \mathrm{Ma}$ vi è di più, perché la posizione di Petrarca riguardo al peccato e alle sue cure si cala in una multiformità avvolgente, dove corpo e anima, morbo e medicina, colpa e penitenza assumono connotazioni prossime e intercambiabili: e il soggetto si trova a svolgere la doppia parte del malato e del medico, o del penitente e del confessore. Anche all'interno di questo linguaggio - il remedium - Petrarca cala il meccanismo di interpretazione polisemica degli amati auctores, così che la cura dell'anima finisce per esser parte di un circuito ermeneutico di fatto inesauribile. Leggiamo qualche riga, scelta fra le più memorabili, sull'uso morale delle fonti - di tutte le fonti, incluse quelle pagane e poetiche- ${ }^{28}$ : quasi una farmacopea

e come calore estivo usa la carità, come rigore invernale il torpore della tentazione, come autunno e primavera la temperanza e discrezione. Sia dunque temperata nel cuore, discreta nelle opere: l'anima che custodisce il contemperamento vive in salute» (da ZOLLA, op. cit., I, p. 545-546).

25. Sulla polemica di Petrarca contro la medicina e la scienza del suo tempo (polemica espressa soprattutto nel De sui ipsius et multorum ignorantia) rinvio soprattutto, in questo volume, a F. P. BОTTI, L'epistola del Ventoso e le misure della rappresentazione petrarchesca della realtà

26. Come è confermato da alcuni luoghi petrarcheschi che rinviano all'ambito della medicina del corpo, come De remediis II 16, 6 («morbo insanabili frustra remedia cogerentur»; o II 35, 8 («morbo ipso peiora remedia»); mentre già sfuma verso il significato spirituale $D e$ remediis II 84, 116: «animorum remedia promisimus, non corporum». Sul valore della parola remedium, centrale soprattutto nell'ambito della filosofia stoica, che fa «de la philosophie la medecine del l'âme», cfr. in particolare il commento di Carraud a Pétrarque, Les remèdes, II p. 150-152.

27. Ha chiarito bene U. DotTI nelle sue note a PÉTrarque, Lettres Familières I-III, Paris: Les Belles Lettres, 2001, p. 398, che "L'image du médicin qui guérit les affliction de l'âme —image che proviént de l'ouvre morale de Cicéron et de Senéque- extrémement fréquente chez Pétrarque». Del resto, remedium era parola usata abitualmente dai confessori nella penitenza, come si evince anche dalle Summe vitiorum et virtutum di Peraldo.

28. Si osservi che anche in Sen. II 1 Petrarca professa l'utilità di ricorrere a fonti classiche (incluso il lascivissimus Ovidio) per avere accesso a una percezione, immanente negli uomini di tutti tempi, del peccato; e che la Sen. IV 5 sostiene l'apertura a ogni interpretazione delle opere che possano sopportarla in misura del loro vero testuale. Sulla polisemia delle fonti nell'esegesi petrarchesca cfr. da ultimo E. FENZI, «L'ermeneutica petrarchesca tra libertà e verità (a proposito di Sen. IV 5)», Lettere Italiane, 54, 2002, p. 170-209 (che a sua volta rinvia, come imprescindibile, a P. C. BORI, L'interpretazione infinita. L'ermeneutica cristiana antica e le sue trasformazioni, Bologna: Il Mulino, 1987). 
«sapienziale» che, alludendo al peccato, fa proprio il linguaggio della cura del corpo. Come Petrarca farà dire a Francesco nel Secretum (II 122):

Consulte quidem; quod ut me non tantum ex philosophicis sed ex poeticis etiam scripturis elicuisse pervideas [...].

\section{Anche Agostino ribadirà di lì a poco la necessità dello stesso remedium:}

Sed, ut unde discesseram revertar, et adversus iram et adversus reliquos motus precipueque adversus hanc, de qua multa iam diu loquimur, pestem, aliquid semper excogita; quod cum intenta tibi ex lectione contigerit, imprime sententiis utilibus (ut incipiens dixeram) certas notas, quibus velut uncis memoria volentes abire contineas (Secretum II 126);

Quotiens legenti salutares se se offerunt sententie, quibus vel excitari sentis animum vel frenari, noli viribus ingenii fidere, sed illas in memorie penetralibus absconde multoque studio tibi familiares effice: ut, quod experti solent medici, quocunque loco vel tempore dilationis impatiens morbus invaserit, habeas velut in animo conscripta remedia. Sunt enum quedam sicut in corporibus humanis sic in animis passiones, in quibus tam mortifera mora est ut, qui distulerit medelam, spem salutis abstulerit. Quis enim ignorat, exempli gratia, esse quosdam motus tam precipites ut, nisi eos in ipsis exordiis ratio frenaverit, animum corpusque et totum hominem perdant, et serum sit quicquid post tempus apponitur? In quibus primum obtinere locum reor iram, cui non frustra rationis sedem superpositam esse diffinunt hi, qui in tres partes animam diviserunt: rationem in capite velut in arce, iram in pectore, concupiscentiam subter precordia collocantes, ut scilicet presto sit, que subiectarum pestium violentes impetus repente coerceat, et ex alto velut receptui canat. Quod frenum, quia ire magis necessarium erat, illi vicinior sita est. (Secretum II 122: mio il corsivo); ${ }^{29}$

Sed [...] et adversus iram et adversus reliquos motus precipueque adversus hanc, de qua multa iam diu loquimur, pestem, aliquid semper excogita; quod cum intenta tibi ex lectione contigerit, imprime sententiis utilibus (ut incipiens dixeram) certas notas, quibus velut unicis memoria volentes abire contineas. Hoc equidem presidio consistes immobilis cum adversus cetera tum contra animi tristitiam (Secretum II 126); ${ }^{30}$

Nec me fallit, ut in corporibus hominum sic in animis multiplici passione affectis, medicamenta verborum multis inefficacia visum iri, sed nec illud quoque me preterit, ut invisibiles animorum morbos, sic invisibilia esse remedia. Falsis oponionibus circumventi veris sententiis liberandi sunt, ut qui audiendo ceciderant, audiendo consurgant (De rem., Pr.).

29. È un brano celeberrimo, nel quale anche A. La PENNA ha riscontrato echi precisi di Orazio, Ep. I 1 e 2 (cfr. Una nota su Petrarca e Orazio, cit.). Si noti in particolare, in tutto il passo, il ricorrere insistente di una terminologia che assimila la cura delle passioni alla medicina del corpo.

30. Sugli uncini della memoria ha scritto pagine importanti M. Feo, Petrarca, in Orazio. cit., 412-414. 
Ma, per quanto «invisibili» siano i remedia, i presidia e i medicamenta dello spirito, per quanto utili a acquisire consapevolezza tramite la sapienza dei classici, o addirittura la poesia dei pagani, essi non corrispondono però alla penitentia sacramentale: piuttosto, prefigurano una cura dell'anima come ricorso a una catena sapienziale e ermeneutica nella quale si collocano anche - ma in certi luoghi solo in forza, parrebbe, di una loro accertata e "filologica" antichità- le Scritture. Rivolgendosi ai monaci che leggeranno il suo De otio (II):

Has ergo Scripturas, quas ego advena necdum notas odore illectus adamavi, sero licet, vos velut indigene et in his a principio enutriti amate, colite, veneramini, frequentate: nusquam de manibus vestris, si possibile sit, certe de mentibus vestris nusquam excidant. Quarum sive auctoritas queritur, et a Spiritu Sancto prolate et Cristi ore firmate sunt, sive antiquitas, et omnes omnino seculares literas antecedunt.

Altra cosa è invece la penitenza cristiana (la sera penitentia o il penitentie rigor del De remediis), ${ }^{31}$ di cui Petrarca dà una lettura ortodossa in De remediis II 126 (De moriente in peccatis), raccomandando il pentimento, anche se tardivo, e esortando il peccatore a evitare la disperazione:

Itaque facta licet infecta esse non possint, ex facto tamen oriens peccatum sic deleri potest, ut uam amplius non sit; ita, ut sicut scriptum est: queratur peccatum et non inveniatur. Non quos se peccati vinculis absolvere potentie sit humane, sed quod pie hominum voluntati contritoque cordi divinum numquam desit auxilium.

Per meglio distinguere i due momenti, potremmo, sintetizzando, affermare che i remedia (gli «uncini» della memoria, le disputationes erudite, il ricorso agli auctores) sono per Petrarca veicolo e strumento di penitenza: in quanto «medicine» essi forniscono all'animo gli antidoti al peccato, chiariscono il dissidio del soggetto, ne accentuano la ratio. Ma — forzando un po' i termini- verrebbe quasi da interpretarli, soprattutto nella loro frammentarietà (nel loro stato primitivo di materiali inerti), anche come sintomi dell'inquietudine del soggetto, del suo incessante oscillare tra dubbio e certezza, fra interrogazione desultoria del passato (i classici) e proiezione verso l'unità della sapienza sub luce eternitatis.

Alla vera e propria penitenza (la satisfactio operis) spetta invece, nell'orizzonte della fede e della pratica cristiana, di sciogliere i nodi del peccato affidando l'uomo alla misericordia di Dio. Ma che in Petrarca i due ambiti semantici siano prossimi, e che, secondo gli orientamenti della catechesi contemporanea, i suoi esercizi spirituali consistano nella ricerca dei remedia e nella aumentata consapevolezza di sé che ne deriva, è del tutto evidente. La via alla sapientia

31. Sulla pratica sacramentale della penitenza al tempo di Petrarca —e specialmente sulle fasi della confessione, sempre più volta all'interiorità della coscienza già a partire dal secolo XIIIcfr. in particolare R. RuSCONI, L'ordine dei peccati. La confessione tra Medioevo ed età moderna, Bologna: Il Mulino, 2002. 
come risultato di un circolo ermeneutico che coivolge tradizione e coscienza ai fini dell'auspicata salute: non c'è da meravigliarsi che al sacerdote-confessore spetti di diritto, come in Fam. X 5, il titolo di medicus.

In questa sinergia - una sinergia che si muove in un orizzonte cristiano, scevro però da rigorismo e radicalismo - si riflette inoltre la profonda convinzione di Petrarca che ogni uomo senta il peso dei propri peccati nell'attimo della morte incombente e a prescindere dalla fede che professa: "Quamvis ergo cui et qualiter confitendum sit nemo nisi cristianus noverit, tamen peccati notitia et conscientie stimulus, penitentia et confessio comunia sunt omnium ratione pollentium» (Sen. II 1). ${ }^{32}$

La voce di Petrarca si unisce al coro degli uomini del suo tempo: recuperandone alcune costanti e insieme propendendo, nella sua sostanza più profon$\mathrm{da}$, verso un'aurorale modernità. Nei confronti di Dante — che possiede una nozione oggettiva della verità a cui ridurre, ecletticamente, gli auctores - per Petrarca verità e sostanza del peccato (e della penitenza) sono invece obiettivi da raggiungere con il cammino dell'intera vita, attraversando tutti i testi per crescere, nella pluralità, assieme a essi. La continua interrogazione - con pari interesse e dignità- della classicità e della tradizione cristiana determina la concrescita del soggetto e la sua progressiva acquisizione della sapientia: il senso autentico della tragicità della condizione umana e della sua colpa non ostacola, ma anzi fomenta, questo circolo protoermeneutico dal quale sia l'autore sia i suoi auctores vengono reciprocamente, progressivamente illuminati. Comprendere ciò significa porre in giusta luce anche il vocabolario della colpa e della pena elaborato da Petrarca: la sua problematicità non significa distacco dalla visione cristiana del peccato, ma la scoperta di un soggettivismo che è il segno di tempi nuovi.

Di certo, rispetto a Dante, che aveva piuttosto guardato al trattato, facendo della Summa di San Tommaso una delle filigrane del Convivio e della Commedia, tra le forme della spiritualità contemporanea Petrarca è più vicino alla confessione, vale a dire all'esplorazione dell'interiorità. La sua non è la confessione che ispira il rituale teologico descritto da Dante nel Purgatorio (IX 70-147), ${ }^{33}$ né men che meno una casistica della colpa, ma un volgersi all'introspezione che assegna rilievo particolare alla contritio cordis e al sentimento «segreto" del peccato. ${ }^{34}$ In ciò Petrarca sembra voler ripercorrere il lungo iter della

32. Si tratta dell'epistola, indirizzata a Boccaccio, con la quale Petrarca ribatte all'accusa dei suoi lettori più critici, che lo rimproverano di avere fatto parlare Magone morente, nell' $A$ frica, con le parole di penitenza di un cristiano.

33. Cfr. in proposito G.G. MEERSSEMAN «Confessione» in Enciclopedia dantesca, cit. II, 1970.

34. E anche in ciò Petrarca è seguace della percezione collettiva del pentimento quale si manifesta fra i suoi contemporanei, che, a partire dagli approfondimenti di teologia morale di Abelardo, Sant'Anselmo, Ugo di San Vittore, avevano posto «reiterato accento sulla coscienza, sull'intenzione, sulla necessità dell'umiliazione interiore» (così J. DELUMEAU, op. cit. p. 360, all'interno di un capitolo dell'opera significativamente intitolato L'esame di coscienza). Sulla stessa lunghezza d'onda si pone M.G. MuZzarelli, Penitenze nel Medioevo. Uomini e modelli a confronto, Bologna: Pàtron, 1994. 
disciplina penitenziale, che proprio verso il XIII secolo si attesta nelle forme dell'esame di coscienza e del "salutare rimorso». ${ }^{35}$ Questa predilezione - a me pare- è enunciata con chiarezza nel dialogo I 46 del De remediis utriusque fortune (De variis titulis studiorum):

Melior sane tutiorque confessio quam professio est. Illa enim humilitatis et penitentie, hec levitatis et insolentie plena est. Multo certe facilius indulgentiam confessi querunt quam scientiam professi,

dove, anche grazie ad Agostino (Conf. V, 5, 8: «Vanitas est enim mundana ista profiteri, pietas autem tibi confiteri»), si chiarisce l'orizzonte di Petrarca riguardo al peccato. Non un pugno di conoscenze dottrinali ma la sapientia — che è tutt'uno con la crescita della soggettività- è strumento e obiettivo di consapevolezza. Ciò vale per tutti gli uomini e per ogni tempo: mentre sono inutili i theologi che "sacrum nomen prophani et loquaces dialectici dehonestant» (De rem. I 46); e permane la certezza che non vi è

nullus [...] fidei articulus, nullum Ecclesie sacramentum, denique nichil evangelicum, nichil omnino quod non in caput hominis multa experti iamque ad finem experientie festinantis secundum naturale ingenium atque insitam rationem possit ascendere [...]. Potest errorem et peccatum suum recognoscere et perinde erubescere ac dolere homo etiam non cristianus, fructu quidem impari, penitentia autem pari (Sen. II, 1).

Come si legge in De remediis I 12 (De sapientia), la sapienza è infatti

inseparabilis a virtute[...]. Non enim res studiis brevis, egens aliquot annorum ut relique artes: tota vel longissima opus est vita [...]. Notum est illud Platonis, ut alia multa, preclarissimum, quod Ciceroni placet et michi: «Beatum cui etiam in senectute contigerit ut sapientiam verasque opiniones assequi possit!».

La sapientia si radica nella classicità, mentre la strada maestra della teologia cristiana riguardo alla colpa si riduce a una formulazione triadica: cognitio, confessio ac penitentia peccati (Sen II 1). In interiore homine si riconosce il peccato; in interiore homine Petrarca va alla scoperta della sua inquieta dimensione autobiografica, richiamando e facendo propria non solo il modello delle Confessiones agostiniane e il sacramento della penitenza ma qualunque fonte possa aumentare la sua cognitio. In estrema sintesi, è la via che lo porta all'analisi del conflitto e al potenziamento dell'introspezione: la via della confessione e della solitudine nella colpa. Non sarà allora un caso che Petrarca non sia mai, a differenza di Dante, membro o spettatore di una schiera di peccatori, ma solo di fronte al baratro che il peccato, individualmente, spalanca. 